\title{
carte postale / deux femmes regardant la mer
}

\author{
Claudine Potvin
}

To go south. To banish the cold. A lovers' paradise. Irene has come to a tropical island with her partner Hubert, a man whose utopia consists of drinking rum and coke, and lying with Irene twenty-four hours a day. Fascinated with Sirens, Irene tells a little girl who sits down beside her on the beach the story of Odysseus who tied himself to his boat in order to resist the enchanting song of the Sirens. When the little girl's mother appears the next day, Irene does not resist. Whereas Hubert transforms Irene into a mermaid-like Siren, her legs tied together inside a sheet, Irene follows the lure of the voices of women, voices capable of re-inventing the world.

j'avais une peau si magnifiquement belle à cause du soleil et de l'excitation comme une rose $[. ..] \mathrm{j}$ 'avais presque envie de me sauver comme une perdue n'importe où nous ne sommes jamais bien où nous sommes [...] où est-elle leur fameuse intelligence je voudrais bien le savoir la matière grise qu'ils ont est dans leur queue voilà ce que je pense $[. .$.$] je suis toujours comme ça au prin-$ temps j'aimerais avoir quelqu'un de nouveau tous les ans (James Joyce, Ulysse)

Allongée sur une plage nonchalante, Irène Lacoste découpe d'un trait la ligne de l'horizon qui se profile dans l'espace assombri de ses lunettes de soleil. Les contradictions de Pénélope s'installent lentement dans son chant. Entre sa vision teintée de la réalité tropicale et ses verres de contact, la beauté des femmes brise le bleu du ciel fait chuter l'absence de nuages que l'huile répandue sur son grand corps blanc moulé dans le sable transparent, aménagé pour la circonstance, accentue. Elle fait l'inventaire de ses jouissances sur ses dix doigts.

Aller dans le sud. Exorciser le froid. Utopie amoureuse. Lieu de causeries conversations inutiles refonte des corps rêveries malaises.

Ils ont fait l'amour ce matin face à la caméra vidéo posée sur le trépied en face du lit, vicieuse, intéressée. Censurer ses appétits. Midi. Plein soleil. Les touristes, fourmis laborieuses en quête de nourriture, exhibent 
l'offre, qui une paire de fesses ballotantes, qui des seins remontés jusqu'à la gorge, qui des muscles d'affaires du Club Méditerranée, qui des sourires blancs de neige. Des femmes passent et repassent dans l'angle droit de l'oeil à demi-clos d'Irène qui repense sa vie en piratesse de l'air. Incendiaire dans l'âme, elle brûle tous ses ponts au nom d'un sauvetage qu'elle sait ridicule mais combien tentant.

La tentation loge sur cette plage, pense-t-elle, hors lieu, ou/topos, tel le fruit qui s'appuie sur le livre ouvert laissant s'écouler le jus sur la page de gauche page vierge non lue. Le papier réchauffé par les quelque trente trois degrés centigrades s'étire, se déforme, acquiert l'odeur et la couleur de la décomposition. Irène ne songe pas à interrompre le modeste flux qui s'initie maintenant entre les lignes de son séjour dans l'île une menstruation venue trop tôt du féminin s'échappe d'elle. Elle pense toujours pouvoir résister à l'appel car elle sait que l'utopie ne fait sens que dans la négation, la dérogation, ce qu'elle appelle l'exil du corps.

Elle n'a rien à dire à ce partenaire enchanté qui s'est déjà installé entre la page et la piscine, entre le bar et le restaurant, entre la couleur locale et l'émerveillement. Perdu dans sa paresse, Hubert avale son sixième rhum \& coke qu'il ne trouve jamais assez fort, pas assez de rhum ou trop de coke. Hubert aime les sensations fortes. Pour lui, le paradis se situe précisément au niveau de la provocation, sur la rampe de l'émotion. Cet homme croyait à la solidité du couple, le chiffre deux roucoulant entre ses cuisses comme une garantie de sa masculinité. Selon lui, les vacances devaient ancrer son désir bien profondément, ou paradoxalement à la surface de ses ébats, au ras de la chaleur et de la transpiration, bonheurs et assurances inclus.

Pour la narratrice, il est presque impossible de penser le personnage Hubert sans l'équivoque, de lui donner une dimension humaine. Le cliché envahit le regard viril, le serre dans ses bras, l'étouffe un peu. Sa respiration avale l'air au fur et à mesure qu'il parle. L'écrire reviendrait à le tuer. Hubert n'échappera pas au stéréotype du meurtre.

Le meurtre n'aura pas lieu ne sera pas absolument nécessaire puisque l'amour se joue en pleine ironie, entre deux phrases, là où l'autre ne perd rien pour attendre. La piste sur laquelle le couple s'engage ne peut que les expulser hors du cercle à la source d'un désir mal entendant.

L'homme et la femme interrompent la conversation pour repartir à zéro. Degré zéro de la tendresse. Entropie. Le couple désamorcé dystrophié en fin de siècle? Ne pas répondre, pas tout de suite. 
Laisser la lumière tracer des gestes sur le long miroir en face du lit. Caméra du silence photographies de l'absence. Courbée sur elle-même, une femme entend la voix d'une baleine fantasmée sur la grève. La mer rejette ses enfants, force centrifuge qui s'exerce dans la foulée des fureurs tropicales qu'Irène ne fait qu'imaginer. Au-delà de cette plage, Montréal s'étend dans le souvenir en redemande invente l'hiver au coin de la rue dévisage les filles décroche la poésie sous les pieds des passantes la ville se précipite sous le repli écumante brisée séduisante française.

Sur le sable froissé, les orteils dessinent des traces le pied enfantin jongle sur les plis assombris par le frisson de l'après-midi se glisse dans le maillot et s'endort. Une main fouille le corps tombé s'appuie sur le bord de la chaise chuchote des mots qui s'apparentent au décor mots d'été capables de lever le voile mots qui encensent mots trompeurs. On dirait l'entente. La fin du jour arrache les amants au prolongement les ramène au pavillon rose de l'hôtel tendu dans sa canicule irréductible les régurgite face à face sans spectacle sans touristes sans enfants. La chambre, Amsterdam ou Marseille, port d'attache, effiloche la représentation de deux corps fatigués, deux bouées de sauvetage coulant sous la lourdeur de la nuit.

Au matin, les corps remontent à la surface n'en peuvent plus de ne pas être heureux. S'amusent, se courent, s'épivardent, se touchent, se baignent, se cherchent dans les vagues, tourbillonnent, se crient des insultes par la tête, s'épuisent à vouloir se plaire, se racontent tous les voyages, toutes les îles, tous les mirages de leur vie d'amoureux, se mangent des yeux, se sèchent, boivent leur bière en silence.

Pour Hubert, vivre en utopie serait vivre tout contre le ventre d'Irène en tout temps vingt-quatre heures par jour ventre refuge paratonnerre creuset pour y loger sa tête de fauve y préparer un amalgame d'épices la route du sucre et du rhum s'y nourrir d'abstractions. Irène refuse de céder son ventre au chercheur d'or.

Au sud il y avait un mouvement que le couple recevait comme une incitation. À tour de rôle, ils tentaient de définir l'objet de leur (mé)contentement mutuel. Hubert nageait dans l'inconnu, à tous points de vue. Ses documents visuels s'amassaient en pile sur la céramique envahissant sa vision de l'île paradis terrestre contenu dans un kilomètre de sable doré et un bain de turquoises s'il lançait une bouteille à la mer, elle lui reviendrait sans doute avec pour tout message le nom des voyageurs.

Viens ici! Viens à nous! Ulysse tant vanté! Arrête ton croiseur: viens 
écouter nos voix! Jamais un noir vaisseau n'a doublé notre cap sans ouïr les doux airs qui sortent de nos lèvres; puis on s'en va content et plus riche en savoir, car nous savons les maux, tous les maux que les dieux, dans les champs de Troade, ont infligés aux gens d'Argos et de Troie, et nous savons aussi tout ce que voit passer la terre nourricière. (Homère, Odyssée, Chant XII)

Au cours de leurs promenades, Hubert explorait le dos bossu de la mer, espérant y déceler une nageoire de requin, savourant d'avance l'excitation du danger, sentant l'adrénaline se précipiter le long des vagues effervescentes. Irène, elle, cherchait des sirènes, entre deux eaux, moitié sauvages, moitié terrestres, une quete de poisson toute de bleu vêtue.

Les sirènes devaient être belles pour tant attirer les marins qui s'approchaient de la côte. Irène se tient indéfiniment face aux vagues, confondant le clapotis avec le chant de ces femmes à la voix d'or. Elle croit les entendre, se retourne, voit bien qu'elles sont toutes là dans leurs costumes rose et bleu, mauve et turquoise, jade ou onyx et qu'elles nagent comme si elles avaient toujours appartenu à l'onde. Elles se balancent sur des flots d'air et de soleil avec des enfants en bandoulière et une odeur d'encens. Elles traversent ce décor lapis-lazuli sur le bout des pieds, en amazones heureuses.

Dans la tête d'Irène, une cascade de sirènes déferle, faisant grand bruit, mélangeant les harmonies du vent à la cacophonie urbaine. À une petite fille venue s'asseoir près d'elle, loin de la surveillance des parents, elle fait le récit de la grandeur de ces femmes centaures.

(Selon la légende, les Sirènes passaient pour dévier le cours des embar cations parties faire la guerre et séduire leurs capitaines. Moi, je prétends que ces enchanteresses, animaux fabuleux à tête et torse de femme et à queue de poisson, se promènent sur la rive, ici et ailleurs, nulle part, indifférentes aux navigateurs et aux écueils qui les guettent. Dans le récit d'Homère, Ulysse, le héros et grand explorateur de cette histoire, boucha les oreilles de ses marins pour qu'ils ne succombent pas au charme des Sirènes. Toutefois, suite aux sages conseils de la magicienne Circé qui avait le pouvoir de changer les hommes en cochons, il se garda bien d'en faire autant de son côté. Il préféra se faire enchaîner au mât de son navire plutôt que de se priver du délice et du plaisir de leurs voix, avisant d'avance ses hommes de ne pas desserrer les liens qui le retenaient même s'il le leur ordonnait.)

(Tu vois, Léane, attaché au poteau, Ulysse fait semblant de résister, 
immunisé dans sa position verticale inchangée, inchangeable. Si le corps et la voix des Sirènes n'entrent pas dans la fiction d'Ulysse, dans mon regard et ma parole, elles jouent de l'accordéon avec tous les ressorts élastiques d'un corps qui s'étire à l'infini et se moquent d'Ulysse car les Sirènes connaissent tous les mots de la terre.)

À cet instant, une mère utopique horizontale au coeur et au corps de sirène surgit de l'océan pour reprendre sa fille et interrompre le récit $\mathrm{d}$ 'Irène.

À cause de sa fascination pour ces ridicules créatures argentées, Hubert compare Irène aux sirènes nocturnes de l'île. La nuit, il lui entoure les jambes avec un drap retenu par les cordons des rideaux de la chambre que plus rien ne retient et s'amuse à lui chercher une vulve où il pourrait s'ébattre. Sans pieds pour s'enfuir, un pubis en forme d'écaille, le sexe cousu fermé refusant les apparences, sans grandes ni petites lèvres, clitérectomisée, buste gonflé par la fiction masculine, Irène/sirène ne peut jouir (que d'elle-même). Il l'inscrit dans le manque, ne lui fabriquant du sens que dans l'oral, la bouche, le son, la voix, le chant, l'impression d'une langue, l'inscrivant dans un dessein faussement animé, pour la bêtise du voyeur. Irène se prête au jeu verbal de l'autre, se mord la langue, épileptique en crises convulsives.

Hubert ne partageait pas l'engouement d'Irène pour les sirènes. Il prend des centaines de photos, la mer à huit heures du matin, placide, en mal d'ouragan, saugrenue, excitée, fanfaronne, la mer au moment où le soleil se penche sur elle pour la baiser toute une nuit durant, les enfants, si rares, admirant les châteaux démolis par la rage et le plaisir de détruire, les hommes et leurs amantes que la dernière marée haute a déversés sur des lits d'algues, fous d'ignorance, désespérés.

Accroché au voilier insouciant, Hubert se balade. Accumule des vidéos de cocotiers, vues de l'hôtel de la plage, vues de la chambre du jardin, vues de la plage du bateau, cartes postales mémoires, carnets de notes, plongées sous-marines, inconsciences. Sous l'eau, une réalité virtuelle promet la fin d'un monde, une sorte de naufrage à l'envers. Hubert semble respirer sous l'eau, siffle sur les poissons qui s'approchent en banc, menaçant de le retenir dans les profondeurs et dans les écrins de corail. Remonter à la surface près d'Irène, croire à la chaleur de son amour.

Des coquillages quittent la grève, s'accumulent sur le lit, dans le bain, dans les cendriers. Ils aboutissent dans le panier en plastique de Léane. La nonchalance s'étend sur les visages de l'homme et de la femme la 
peau se métamorphose les énergies se vident une sensation d'ennui perdure là où la lumière du jour vient chercher le regard envahissant de l'autre faim de silence et de paix. À la fin, il ne reste plus qu'à regarder la mer.

Irène s'éloigne. À l'aube, elle s'enroule dans sa serviette, se compose une façade de mer du Maine, s'enfonce dans l'eau et se laisse glisser dans l'extase savoure sa solitude prête à bondir sur la dernière venue.

Je disais, pense la narratrice, avec dans la bouche un goût de sel, à propos de l'utopie en commençant par le mot femme que l'utopie n'allait pas assurer notre insertion dans la réalité mais qu'un témoignage utopique de notre part pouvait stimuler une qualité d'émotion propice à notre insertion dans l'histoire. (Nicole Brossard, Picture Theory)

Le lendemain, la mère est remontée jusqu'à elle, sans l'enfant, pour jaser. Murmures liquides. Sont devenues amantes d'eau d'une huître à l'autre se sont faites moules ont répandu du vin sur leurs rires mis et remis «Le parapluie», fredonné leur désir de continuer en se tenant par la taille. Des heures de sommeil, des étendues de palmiers tout croches, des fruits de mer marinés cuits sur le gril, des petits creux dans le sable, une fossette au coin des lèvres, une peau tendre, juteuse, tendue, une toile de parasol bariolée, impudique, et la joie des Tropiques. Utopiques.

Sur le rivage, le champ solaire s'étend à n'en plus finir, rejoint les citadins momentanément déplacés, angoissés dans l'ombre vaine de leurs attentes. Les deux femmes marchent au-devant d'elles-mêmes, audevant du bonheur d'être, indifférentes à la prise du temps chaud sur leur épiderme. Plongées dans le temps présent qui peut tout bouleverser, l'embarcation, la rive, le capitaine, la douceur, l'illusion, le savoir, les mathématiques. Deux femmes dans l'utopie de la langue, sous l'effet du sens, dans la foulée d'un climat idylle généreux surexcité abondant. À se frotter sur le sable mouillé à la dérive des mots parachutés les uns sur les autres des mots au corps léger pleins de rebondissements refusant de se taire des mots farfelus bouffons se repliant sur l'étreinte et l'avant-bras de celle qui tend la poitrine. Les jambes s'étirent sur les rochers. Deux femmes se plient aux exigences du jour caracolent repensent le sens du voyage s'adonnent à l'exil se parlent de la vie des autres de l'entraînement à l'indifférence du temps qu'il fait de la contrainte des heures. Substituts. 
Irène abolit la distance entre sa matrice, le col de son utérus et sa langue. Elle se dit des choses à son oreille et se gonfle d'orgueil, laissant ses doigts tambouriner sur son ventre à l'affût du moindre signe. Tout son corps signifie dans la liquidité qui l'absorbe. Fluidité de la découverte. De l'espace, du chant des sirènes, auquel elle ne songe pas à résister. Charmée, elle s'appuie sur la coque et fait semblant de se laisser aller dans l'eau qui fouette timidement le navire. Le sud lui redonne le goût de jouer comme autrefois la petite fille au bord du lac mais elle sait que le rêve utopique ne revient pas sur le passé l'enfance est une fausse piste l'enchantement ne peut s'étaler que dans le présent, dans le tourbillon, loin des illusions, loin des cadres sclérosés accrochés aux musées anciens poussiéreux, d'échapper aux regards obscènes.

La mère s'entend dire qu'ici le soleil se lève toujours à la même heure que les femmes sortent tôt pour se rendre au marché que la beauté s'affiche pour rien qu'il faut savoir aimer jusqu'à la démesure jusqu'au tourment.

Irène Lacoste ravale ses inquiétudes s'enfonce à perte de rêves s'éprend de ses amours toutes neuves fringantes avale la chair de crabe sensuelle pâle attentive au moindre soupir. Le sexe se fraie un chemin dans sa gorge ivre de tant de découvertes. Elle s'acotte sur sa compagne. Sur la côte, un rocher perd le nord, effaçant toute raison d'être, toute justification. Seuls subsistent les jeux inscrits dans la pierre noire polie par les habitants de l'île qui ne s'étonnent plus de rien, surtout pas de deux femmes amoureuses regardant la mer, embrassant l'éclat du paysage qui surgit inopinément dans l'oeil de la narratrice sans qu'elle n'y puisse rien.

Tableau dirigé sur les premières femmes, Èves perverses, frivoles, nues, curieuses, paresseuses, jalouses. Contemplatives en mutation, explosives. Hors d'un Éden pensé ailleurs, envahi par la foudre intimiste et les orages intérieurs. Les amantes refusent l'installation. La narratrice écrit que l'épanchement des corps suppose un amour fou des mots, une quête d'un langage qui saurait décortiquer un sentiment complexe, obscur, qui se dirait en termes de désirs du coeur et du corps. Irène accoste de l'autre côté du réel n'en attendait pas moins de ce pôle d'attraction s'étonne de se savoir au bon endroit au bon moment cruise sans vergogne. Tous les chemins mènent à la mère.

Retour aux sirènes. Elles circulent dans la pensée d'Irène. Logent dans les noms. S'aventurent. Toujours plus près des visiteurs. Runaway Bay, 
Playa del Carmen, Isla Mujeres, Acapulco, Varadero, Santa María del Mar, Ocho Ríos, Negril, Turtle Bay, Trinidad, Pointe-au-Pic. Irène embrase la rondeur du sud contenue dans les langues familières de cette cartographie lectures parcours d'un territoire immensément humain d'une humanité générique qui commencerait avec une mère/amante.

Léane suit les deux femmes comme si elles n'étaient plus qu'une seule mère débordante d'affection pour la fillette abasourdie de tant d'attentions. Les vacances de Léane renversent les limites du quotidien. Ne comprend pas tout, échafaude une fiction dans laquelle les hérö̈nes, des sirènes, réinventent le monde, s'embrassent sous le feu des brasiers, imaginent quelque chose de doux, des chattes d'une langueur indicible.

De temps en temps, elle abandonne ses séductrices aux seins découverts et rejoint Hubert isolé sur son île. Hubert attend le retour d'Irène et, dans sa tête, il lui promet mondes et merveilles. L'enfant lui explique l'amour mais l'homme pense à autre chose. 